\title{
Fast Design Space Exploration of Vibration-Based Energy Harvesting Wireless Sensors
}

\author{
Tom J. Kazmierski, Senior Member, IEEE, Leran Wang, Member, IEEE, Geoff Merrett, Member, IEEE, Bashir M. \\ Al-Hashimi, Fellow, IEEE, Mansour Aloufi, Member, IEEE
}

\begin{abstract}
An energy-harvester-powered wireless sensor node is a complicated system with many design parameters. To investigate the various trade-offs among these parameters, it is desirable to explore the multi-dimensional design space quickly. However, due to the large number of parameters and costly simulation CPU times, it is often difficult or even impossible to explore the design space via simulation. This paper presents a response surface model (RSM) based technique for fast design space exploration of a complete wireless sensor node powered by a tunable energy harvester. As a proof of concept, a software toolkit has been developed which implements the proposed design flow and incorporates either real data or parametrized models of the vibration source, the energy harvester, tuning controller and wireless sensor node. Several test scenarios are considered, which illustrate how the proposed approach permits the designer to adjust a wide range of system parameters and evaluate the effect almost instantly but still with high accuracy. In the developed toolkit, the estimated CPU time of one RSM estimation is $25 \mu \mathrm{s}$ and the average RSM estimation error is less than $\mathbf{1 6 . 5}$
\end{abstract}

Index Terms-Tunable energy harvester, wireless sensor node, hardware description language, response surface model, simulation.

\section{INTRODUCTION}

Wireless sensors powered by kinetic energy harvesters are extremely complex systems, operating in multiple technology domains: analog electrical, digital, mechanical and magnetic. Energy harvesters, specifically tunable energy harvesters, which can adjust their own resonant frequency to match the input frequency, are deemed to be a suitable power source for wireless sensor nodes [1]. Energy supply issues in wireless sensor networks (WSNs) are becoming more and more important to both industry and academia, due to many emerging applications such as environmental sensing [2], structural monitoring [3] and pervasive healthcare [4]. Optimal design of such systems is a difficult problem due to the need to optimize the entire sensor node holistically [5] and prohibitive simulation times resulting from the complexity.

To simulate energy harvester sensor nodes efficiently, models based on hardware description languages (HDL), such as VHDL-AMS and SystemC-A have recently been proposed $[5,6]$. However, these solutions have not fully solved the main challenge of the traditional analog simulation approach based on Newton-Raphson iterations which lead to long CPU times. Even though we have developed a linearized state-space

The Authors are with the Faculty of Physical Sciences and Engineering, University of Southampton, Southampton, SO17 1BJ, UK, (phone: +44 2380593520; fax: +44 2380592901; email: \{tjk,lw04r,gvm,bmah,ma08r\}@ecs.soton.ac.uk) technique which can reduce the CPU time of one simulation by two orders of magnitude [7], it is still not feasible to optimize the energy performance of a complete wireless sensor node where many thousands of simulations are required. Also, although classical multi-variable optimization methods based on gradient searches and various heuristic algorithms, such as genetic optimization or simulated annealing, allow improvements to an initial design, they do not lend themselves easily to fast design space exploration and investigation of possible trade-offs. This paper addresses the issue of fast design exploration in a complex design space and proposes a technique which is based on response surface models (RSM), a common design of experiments (DoE) approach in other fields of science and engineering. After corresponding RSM models of each performance characteristic are built, the search of the design space is almost instant. We have developed a toolkit to demonstrate the efficiency of this technique.

DoE was first adopted as a formal approach in the early 1920s in agricultural, experimental research [8]. Nowadays, DoE approaches are common practice in industrial and scientific experimentation. This research has focused on combining DoE with HDL-based modeling of a complex energyharvesting sensor node to enable designers to explore the available design space very quickly, without the need for costly simulations. A moderate number of simulations is required initially to build the RSMs, but once the design space is approximated and captured, its exploration is very fast.

In this paper we propose to use a set of RSMs as a means of fast exploration of the design space where a number of sensor node performance indicators can be evaluated very quickly as functions of selected design parameters. To maximize the accuracy of the response surface models, we have adopted the D-optimality criterion [9] in calculating the design points on which the RSMs are built. Full simulations are carried out for each of the design points to obtain the performance indicator values from which the RSM coefficients are obtained. Although the number of the design points is moderate, from 21 to 78 in our test scenarios, the use of the fast linearized state-space technique [7] allows the RSMs to be built in less than 5 hours. Once the RSMs are built, exploration of the design space and investigation of trade-offs between the design parameters and performance indicators is instantaneous.

The paper is organized as follows. Section II outlines the principles of our approach. Section III describes the proposed design space exploration technique while Section IV presents the developed toolkit. Section V shows the results from several test scenarios and Section VI draws conclusions and identifies 
areas of future work.

\section{PROPOSED DESIGN FLOW}

Fig. 1(b) outlines the design flow proposed in this paper. Firstly, an HDL model of the entire, energy-harvester powered wireless sensor node is created. Secondly, once the design parameters and their ranges are determined, a number of simulations are run at the design points determined by the Doptimal algorithm (Section III) to build a set of the response surface models for the desired performance parameters.

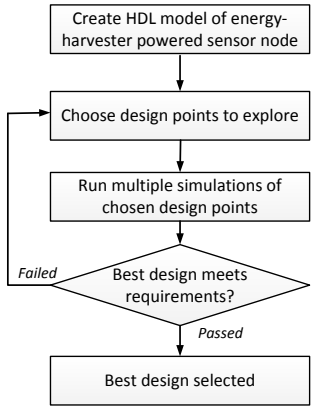

(a)

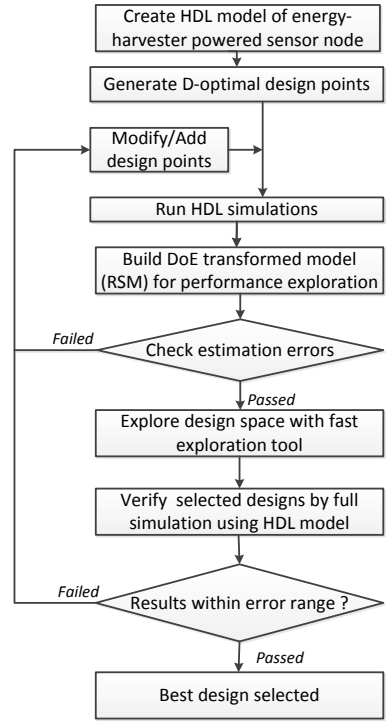

(b)
Fig. 1. Comparison of work flows for design exploration of energy harvesting sensor nodes; (a) Traditional design flow using multiple simulations; (b) Proposed design flow using RSM based fast design exploration.

This method provides detailed relationships between the design parameters and performance indicators. The process of building an RSM removes the detail by approximating these relationships with multivariate polynomials. Typical design parameters in the complete wireless sensor node shown in Fig. 2 may include the mechanical microgenerator proof mass, its Q-factor, storage size, power processing circuit configurations, etc. A suitable set of performance parameters, calculated from simulation results, is selected to provide quality metrics of the design. For example, it is desirable to maximize the power generated by the microgenerator, while minimizing the enery lost at the power processing circuit. At the energy consumption end, it is crucial to adjust the duty cycles of the sensor node according to the available energy so that the maximum amount of information can be gathered and transmitted without depleting the energy source. A number of trade-offs exist between the design parameters and the extent to which they affect performance. It is clear that for such a complicated system to work efficiently, many design parameters need to be chosen collectively due to these trade-offs. For example, the tuning controller wakes up periodically to check if the microgenerator's resonant frequency matches the input vibration frequency. An increase in the tuning controller duty cycle will reduce the energy consumption but the longer duty cycle means that the response to input frequency change will be slower and therefore less energy can be generated. For a designer, it is necessary to investigate these tradeoffs and to explore the design space. Hardware description languages have been employed for this purpose in recent years. However, HDL-based design space exploration for the application discussed here is inefficient due to prohibitive CPU times. Table I illustrates this problem. If the CPU time $T$ required for one simulation is say, 3 hours, and the number of simulations $N$ required to explore the design space in sufficient detail is 500, the whole process would take 1500 hours. On the other hand, with $k=8$ design parameters, the number of simulations required to build a set of RSMs is only $n=45$ and, additionally, the simulations can be carried out in parallel on a computer cluster as the design points at which the design is simulated are reselected in advance by the Doptimal algorithm. Once the RSMs are built, arbitrary designs can be explored within the design space covered by the RSM and their performance evaluated instantly.

\section{A. System model}

To model a complete wireless sensor node system powered by a tunable energy harvester (Fig. 2) we have incorporated both physical, i.e. mechanical and magnetic, as well as geometric parameters of the microgenerator, circuit-level parameters of the analog electronic components, digital processes that implement the underlying control algorithms, and interactions between the different physical domains. Additionally, the energy consumption of the system components has been modeled as equivalent variable resistors, whose values are dynamically changing and linked with the control scenarios executed by the digital processes. To demonstrate the proposed approach, we use the available HDL set of models that describe a complete wireless sensor node which exhibits complex interactions between blocks and is modeled on a variety abstraction levels in the electrical, magnetic and mechanical physical domains [5]. To illustrate the model's complexity and demonstrate the proposed design space exploration approach, below we present the model of the tunable microgenerator as an example.

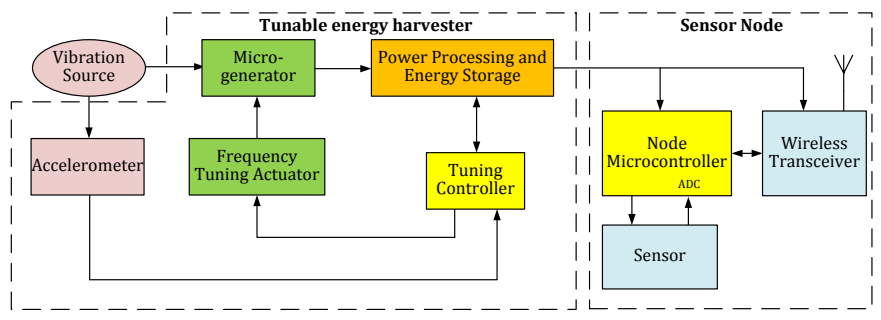

Fig. 2. Key components of a wireless sensor node with a tunable energy harvester.

Fig. 3 shows the arrangement of the electromagnetic microgenerator and its tuning mechanism. The microgenerator is based on a cantilever structure. The coil is fixed to the base, and four magnets (which are located on both sides of the coil) form the proof mass. The tuning mechanism uses magnetic 
TABLE I

CPU TIMES OF THE TRADITIONAL AND PROPOSED DESIGN SPACE EXPLORATION TECHNIQUE

\begin{tabular}{|l|l|l|l|l|l|}
\hline $\begin{array}{l}\text { System } \\
\text { model }\end{array}$ & $\begin{array}{l}\text { CPU time of } \\
\text { one simulation }\end{array}$ & $\begin{array}{l}\text { No of } \\
\text { parameters }\end{array}$ & $\begin{array}{l}\text { No of simulations } \\
\text { to build RSM }\end{array}$ & $\begin{array}{l}\text { CPU time of } \\
\text { RSM estimation }\end{array}$ & $\begin{array}{l}\text { CPU time of evaluating } \\
N \text { design points }\end{array}$ \\
\hline $\begin{array}{l}\text { Traditional design } \\
\text { space exploration }\end{array}$ & $T$ & & & $N * T$ \\
\hline $\begin{array}{l}\text { Proposed design } \\
\text { space exploration (Section III) }\end{array}$ & $T$ & $\mathrm{k}$ & $n=1+2 k+C_{k}^{2}$ & $25 \mu \mathrm{s}$ & $n * T+N * 25 \mu \mathrm{s}$ \\
\hline $\begin{array}{l}\text { Sample scenario: Ferry vibrations with } \\
\text { SBC power processing (Section V) }\end{array}$ & $T=292$ mins & $k=8$ & $n=45$ & $25 \mu \mathrm{s}$ & Traditional: $N * 292 \mathrm{mins}$ \\
\cline { 2 - 6 } & & & & \\
\hline
\end{tabular}

force to change the effective stiffness of the cantilever which leads to a change of resonant frequency. One tuning magnet is attached to the end of the cantilever beam and the other tuning magnet is connected to a linear actuator. The linear actuator moves the magnet to the calculated desired position so that the resonant frequency of the microgenerator matches the dominant frequency of the ambient vibration.

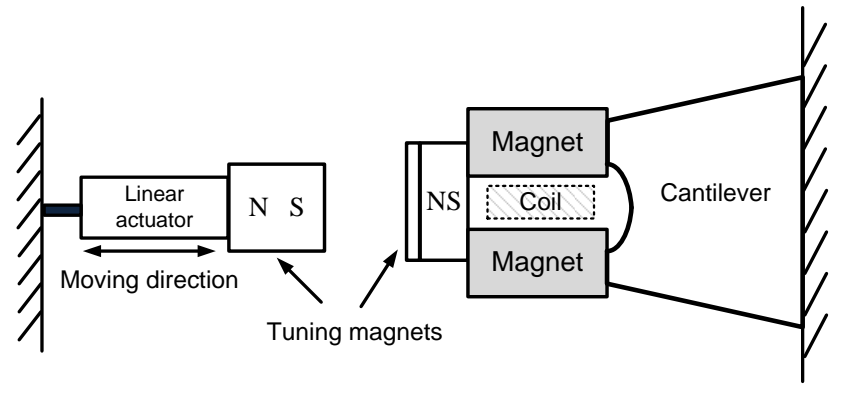

(a) Top view.

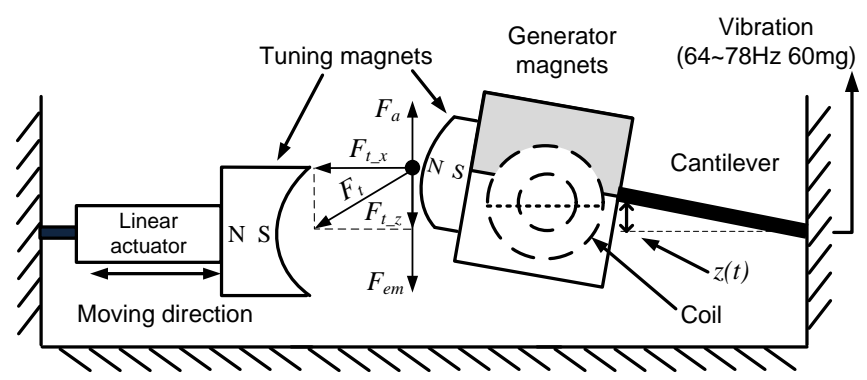

Fig. 3. Diagram of the tunable (b) Side view.

Fig. 3. Diagram of the tunable electromagnetic microgenerator showing its components and tuning forces.

The dynamic model of the microgenerator is [10]:

$$
m \frac{\mathrm{d}^{2} z(t)}{\mathrm{d} t^{2}}+c_{p} \frac{\mathrm{d} z(t)}{\mathrm{d} t}+k_{s} z(t)+F_{e m}+F_{t, z}=F_{a}
$$

where $m$ is the equivalent vibrating mass, $z(t)$ is the relative displacement between the mass and the base, $c_{p}$ is the parasitic damping factor, $k_{s}$ is the effective spring stiffness, $F_{e m}$ is the electromagnetic force, $F_{t, z}$ is the $z$ component of tuning force $F_{t}$, and $F_{a}$ is the input acceleration force. The $z$ component of tuning force is:

$$
F_{t, z}=F_{t} \frac{z(t)}{l_{c}}
$$

where $l_{c}$ is the length of the cantilever.
The resonant frequency $\omega_{0}$ and damping coefficient $\zeta$ are:

$$
\begin{aligned}
\omega_{0} & =\sqrt{\frac{k_{s}}{m}} \\
\zeta & =\frac{c_{p}}{2 \sqrt{m k_{s}}}
\end{aligned}
$$

Fig. 3 also shows all the forces acting on the generator. $F_{t, z}$ represents the $z$ component of the tuning force. It is typically omitted in the dynamic equation of the tunable microgenerator [10]. It is normally assumed that when the two tuning magnets are far apart, the tuning force is small and its $z$ component is negligible. However, we found that simulation results obtained without $F_{t, z}$ differ from experimental measurements when the two tuning magnets are closing together, i.e. the tuned resonant frequency is increasing, because the tuning force is becoming larger and its $z$ component begins to affect the microgenerator's behavior. The $z$ component of the tuning force has therefore been included in equation (1) to ensure the accuracy of the model.

As the two tuning magnets can be approximately treated as regular cuboids, the method developed by Akoun and Yonnet [11] can be adopted here to calculate $F_{t}$. For two cuboid magnets sharing the same central line along their thickness and with the area where these two magnets face each other, as shown in Fig. 4, the magnetic force between them is [10]:

$$
\begin{aligned}
& F_{t}=\frac{M_{1} \cdot M_{2}}{4 \pi \mu_{0}} \sum_{i=0}^{1} \sum_{j=0}^{1} \sum_{k=0}^{1} \\
& \sum_{l=0}^{1} \sum_{p=0}^{1} \sum_{q=0}^{1}(-1)^{i+j+k+l+p+q} \phi\left(u_{i j}, v_{k l}, w_{p q}, r\right)
\end{aligned}
$$

where $M_{1}$ and $M_{2}$ are the magnetization of these two magnets, $\mu_{0}$ is the magnetic constant and $\phi\left(u_{i j}, v_{k l}, w_{p q}, r\right)$ is a function of dimensions of the two magnets and their relative position. For the magnet configuration shown in Fig. 4, the interactive force between the two magnets is parallel with their polarization and $\phi$ is given by:

$$
\begin{aligned}
& \phi\left(u_{i j}, v_{k l}, w_{p q}, r\right)=-u_{i j} \cdot w_{p q} \cdot \ln \left(r-u_{i j}\right)-v_{k l} \cdot w_{p q} \\
& \cdot \ln \left(r-v_{k l}\right)+u_{i j} \cdot v_{k l} \cdot \tan ^{-1} \frac{u_{i j} \cdot v_{k l}}{r \cdot w_{p q}}-r \cdot w_{p q}
\end{aligned}
$$

where $u_{i j}=(-1)^{j} \cdot H-(-1)^{i} \cdot h, v_{k l}=(-1)^{l} \cdot W-$ $(-1)^{k} \cdot w, w_{p q}=d+\frac{L+l}{2}+(-1)^{q} \cdot L-(-1)^{p} \cdot l$ and $r=\sqrt{u_{i j}^{2}+v_{k l}^{2}+w_{p q}^{2}}$. 


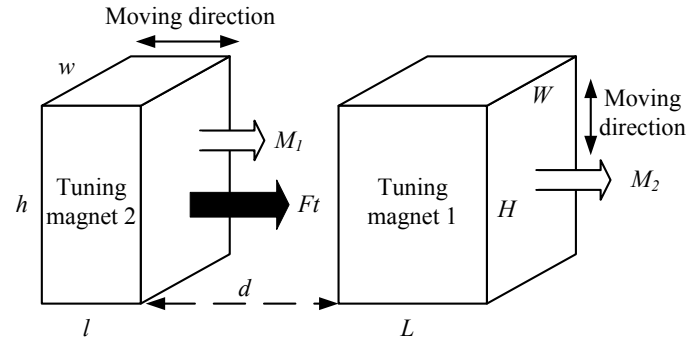

Fig. 4. Configuration and parameters of the two tuning magnets.

The resonant frequency of the tuned microgenerator $\left(f_{r}^{\prime}\right)$ is:

$$
f_{r}^{\prime}=f_{r} \sqrt{1+\frac{F_{t}}{F_{b}}}
$$

where $f_{r}$ is the un-tuned resonant frequency, $F_{t}$ is the tuning force between two magnets and $F_{b}$ is the buckling load of the cantilever.

The electromagnetic voltage generated in the coil is:

$$
V_{e m}=-\Phi \frac{\mathrm{d} z(t)}{\mathrm{d} t}
$$

where $\Phi=N B l$ is the transformation factor and $N$ is the number of coil turns, $B$ is the magnetic flux density and $l$ is the effective length. The output voltage is:

$$
V_{m}(t)=V_{e m}-R_{c} i_{c}(t)-L_{c} \frac{\mathrm{d} i_{L}(t)}{\mathrm{d} t}
$$

where $R_{c}$ and $L_{c}$ are the resistance and inductance of the coil respectively and $i_{c}(t)$ is the current through the coil. The electromagnetic force is calculated as:

$$
F_{\text {em }}=\Phi i_{c}(t)
$$

Typical design parameters of the microgenerator presented and the associated tuning control algorithm can be: the proof mass $M$, number of coil turns $N$, mechanical $Q$-factor and tuning control duty cycle $D_{t}$. Associated performance indicators are calculated from the accurate model which uses the above equations (1-10) but once corresponding RSMs are built, they can be estimated very fast from exploration of the approximation design space. Suitable performance indicators may include: the generated power, maximum displacement of the vibrating magnet and power consumption of the tuning control. In addition, design parameters associated with the other components of the sensor node, i.e. the power processing circuit, storage element and sensor node, as well as their corresponding performance indicators, can be added to the fast design space exploration model as explained in the following sections.

\section{FAST DESIGN SPACE EXPLORATION}

We have adopted the response surface modeling (RSM) methodology [9] to build multi-variable polynomial approximations of the relationships between the energy harvester design parameters and required performance indicators. Once RSM models are constructed, they can be evaluated very fast, in real time, such that designers can obtain instant answers concerning trade-offs between design parameters and performance characteristics. The formation of an approximated mathematical model is carried out by fitting the results obtained from a series of simulations that cover the design space of interest with the polynomial coefficients of the RSM model using regression analysis. It has to be acknowledged that the RSM model represents only an approximation and the final set of energy harvester parameters selected by the designer should be verified by a full simulation. However, the value of the RSM approach lies in its speed and ease of use thus allowing designers to develop knowledge and understanding of complex relationships between design parameters and performance which would otherwise be either impossible to obtain, or take a very long time.

Briefly, the RSM technique can be outlined as follows. Suppose performance indicators $y \in R^{r}$, where $r$ is the number of performance indicators, is affected by $k$ design parameters $a \in R^{k}$. Let the relationship between $y$ and $a$ be approximated as:

$$
y=f\left(a_{1}, a_{2}, \ldots, a_{k}\right)+\epsilon
$$

where $\epsilon$ represents the approximation errors, $a_{1}, a_{2}, \ldots, a_{k}$ are the design parameters and $f()$ are the approximation functions that relate the design parameters with the performance indicator.

In most practical applications the approximation functions $f()$ are low-order polynomials or multi-dimensional splines. The design parameters $a_{1}, a_{2}, \ldots, a_{k}$, which are expressed in their corresponding physical units, must be converted to dimensionless, normalized quantities with zero mean and the same standard deviation for the purpose of RSM analysis and regression. The new quantities are called coded variables (i.e $\left.x_{1}, x_{2}, \ldots, x_{k}\right)$ and the transformation process is achieved by normalizing $a_{k}$ between $[-1,1]$ with zero mean.

Now the approximation functions are expressed in terms of the coded variables $\left(x_{1}, x_{2}, \ldots, x_{k}\right)$ as follows:

$$
\hat{y}=\beta_{0}+\sum_{i=1}^{k} \beta_{i} x_{i}+\sum_{i=1}^{k} \beta_{i i} x_{i}^{2}+\sum \sum_{i<j} \beta_{i j} x_{i} x_{j}
$$

where $\beta_{0}, \beta_{i}, \beta_{i i}, \beta_{i j}$ are the coefficients of the intercept, linear, quadratic and interaction in the regression model respectively, $x_{i}, x_{j}$ are the design parameters in their coded format. This is called the response surface model. The coefficients of the polynomial in equation (12) are determined through $n$ simulation runs of the detailed, energy harvester model in an HDL. The number of simulation runs $n$ necessary to obtain the polynomial coefficients in equation (12) is $n=1+2 k+C_{k}^{2}$, where $C_{k}^{2}=\frac{k(k-1)}{2}$. The design points of the $n$ runs are determined using the D-Optimal criterion as explained later in this section. Using matrix notation, equation (12) can be written as:

$$
\hat{\boldsymbol{y}}=\boldsymbol{X} \beta
$$

where $\boldsymbol{X}_{r \times p}$ is $r \times p$ design matrix. The difference between the observed values $\boldsymbol{y}$ and fitted values $\hat{\boldsymbol{y}}$ for the $i$ th observation $\epsilon_{i}=y_{i}-\hat{y}_{i}$ is called the residual for that specific observation. The coefficients $\beta$ are chosen such that the resulted sum of the residual squares (SSE) is minimised. 
It may seem strange that such a simple, polynomial approximation should be sufficient to represent the behavior of a very complex energy-harvester-powered sensor node whose detailed, circuit-level model is constructed using physical equations from different technology domains combined with algorithms executed by microcontrollers. However, for the purpose of performance exploration, much of the inner detail is lost when it comes to the relationships between design parameters and typical performance indicators, such as the generated power or number of data transmissions. This loss of inner detail is taken advantage of when constructing the RSM approximation.

The choice of design points at which the system is simulated for the purpose of building the RSM is selected by the DOptimal strategy [9]

\section{DESIGN EXPLORATION TOOL}

The design space of the system in Fig. 2 contains design parameters from each building block. Some of the parameters are continuous and some are discrete (such as the different circuit topologies). In each block, we listed below the design parameters and the performance indicators included in the developed design exploration tool. Fig. 5 shows a screenshot of the developed tool which is a front-end providing easy exploration of the RSM.

a) Vibration source: The vibration source can be considered as either real vibration data (obtained from practical experiments), or a sine wave. The user parameters are:

- Sine wave vibration with varying frequency and amplitude $\left(5-10 \mathrm{~m} / \mathrm{s}^{2}\right)$. The frequency jumps between $50 \mathrm{~Hz}$ and $55 \mathrm{~Hz}$. The user can set the time between each frequency change (30-50mins).

- Real vibration data collected from a ferry [12]. The ferry travels between two locations back and forth. Fig. 6(a) shows the vibration characteristics over 200 minutes (the time taken for an outward and return voyage). When the ferry is traveling, the vibration level is high and the dominant frequency is almost constant with slight variations around $50 \mathrm{~Hz}$. When the ferry is docking, there is no significant amount of vibration and there is no dominant frequency.

- Real vibration data collected from a car engine [12]. The 45-minute drive covers both city center roads and motorways. Unlike the ferry boat which runs a fixedspeed engine, the car engine generates a much wider frequency spectrum and the dominant frequency also varies rapidly (Fig. 6(b)). Both narrow-band and wideband real vibration data are included in our tool to investigate their effects on energy harvester designs.

b) Microgenerator: The microgenerator is a tunable electromagnetic system.Although the general theories behind electromagnetic microgenerators are the same, the actual device structures are varying. We have included some most common and important user parameters in the tool and these are:

- Proof mass $(5-15 \mathrm{~g})$

- Mechanical Q factor (100-300)

- Number of turns in the coil $(1000-5,000)$

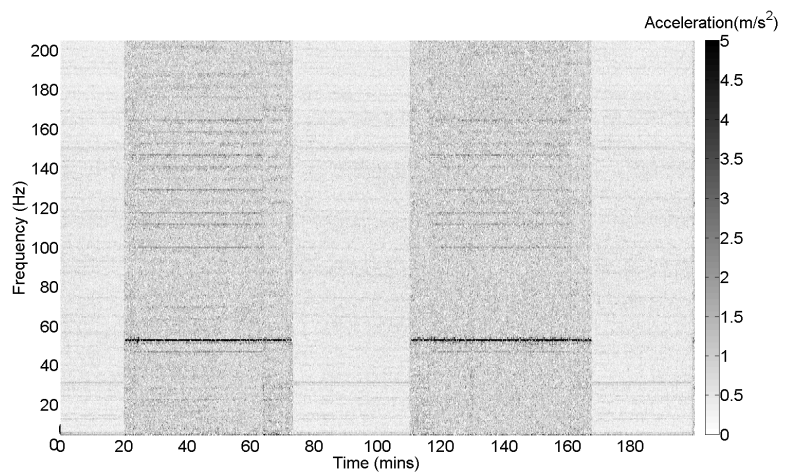

(a) Ferry vibration

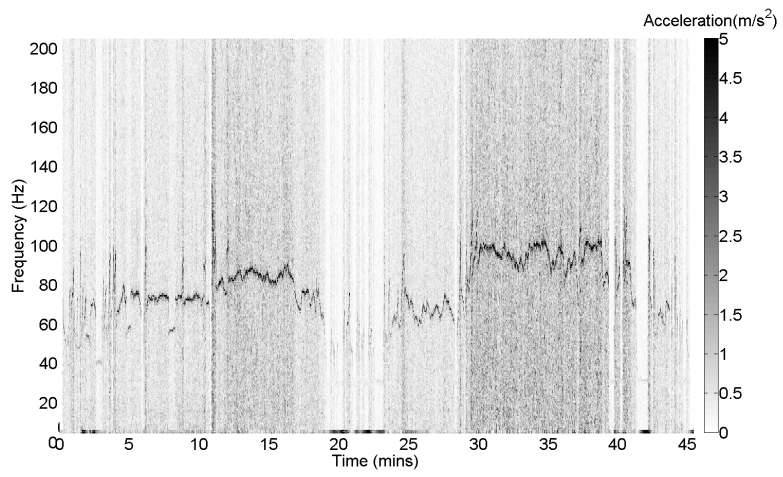

(b) Car engine vibration

Fig. 6. Frequency characteristics of real vibration data.

The performance indicators are the generated power and maximum displacement of the proof mass.

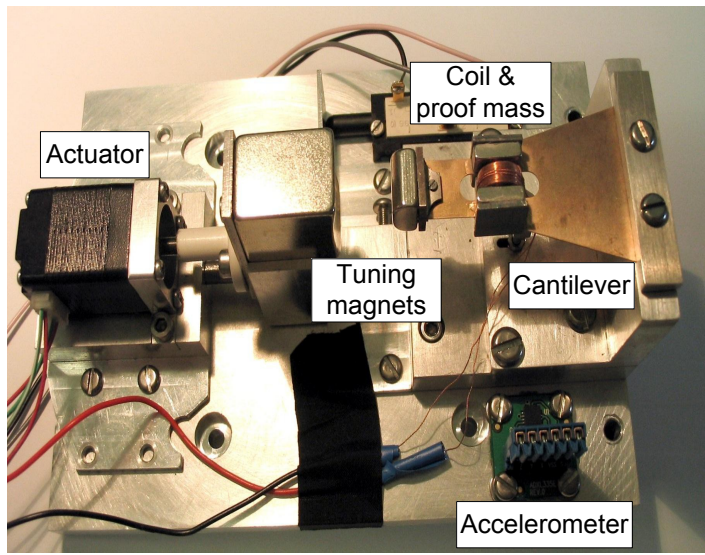

Fig. 7. Photo of a prototype tunable energy harvester.

c) Power processing: Three types of power processing circuits have been chosen as:

- Bridge rectifier (BR) (Fig. 8(a))

- 4-stage voltage multiplier (VM) (Fig. 8(b))

- Switching boost converter (SBC) with varying inductor value $(1-10 \mathrm{mH})$, switching frequency $(10-50 \mathrm{kHz})$ and switching duty cycle (20\%-90\%) (Fig. 8(c))

The performance indicators are energy consumption and transfer efficiency. 


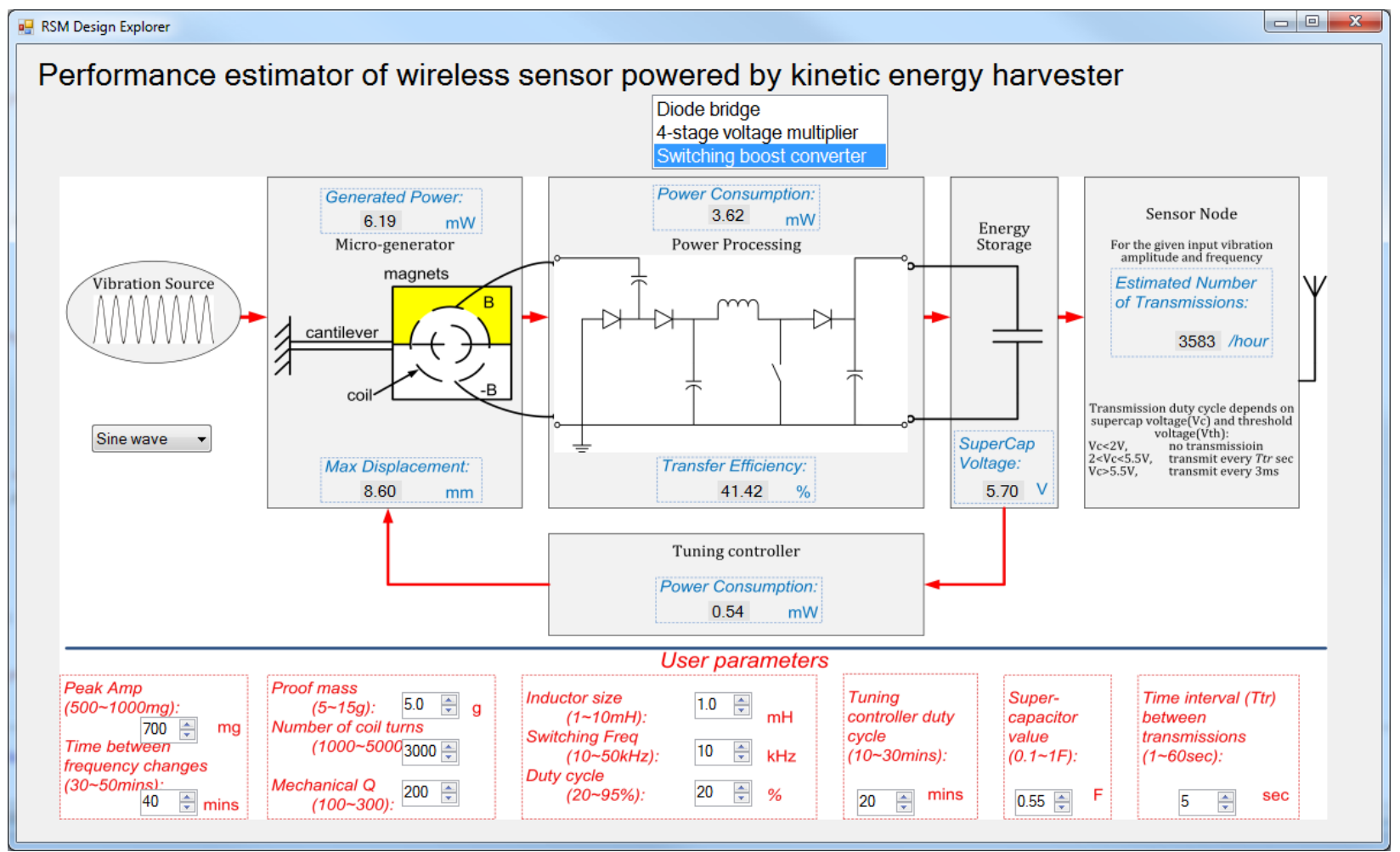

Fig. 5. Screenshot of the design exploration tool showing the performance metrics in the top half of the screen (e.g. transfer efficiency and number of transmissions) and design parameters at the bottom of the screen (e.g. proof mass, inductor size etc)..

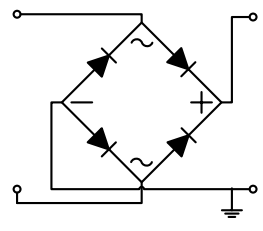

(a) Bridge rectifier

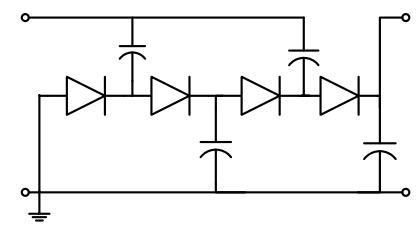

(b) 4-stage VM

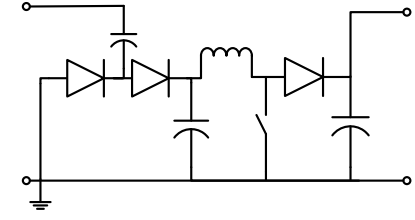

(c) Switching boost converter
Fig. 8. Power processing circuit topologies.

d) Energy storage: The design parameter is the supercapacitor value $(100-1000 \mathrm{mF})$ and the performance indicator is the supercapacitor voltage.

e) Sensor node: Software on the sensor node's microcontroller configures the node in an energy-aware manner, by adjusting its transmission interval $\left(T_{t r}\right)$ in response to the energy available in the supercapacitor. This operation is summarized in Table II. The design parameter is the transmission interval. The performance indicator is the number of transmissions per minute.

f) Tuning control: The tuning control includes the tuning controller, the accelerometer and the tuning actuator. The
TABLE II

SENSOR NODE BEHAVIOR AS A FUNCTION OF SUPERCAPACITOR VOLTAGE

\begin{tabular}{|l|l|}
\hline Supercapacitor voltage & Node behavior \\
\hline Below $2.0 \mathrm{~V}$ & Wake up every 1 minute, no transmission \\
\hline Between 2.0 and $5.5 \mathrm{~V}$ & Wake up and transmit every $T_{t r}$ seconds \\
\hline Above $5.5 \mathrm{~V}$ & Wake up and transmit every $3 \mathrm{~ms}$ \\
\hline
\end{tabular}

design parameter here is the tuning controller duty cycle (1030mins) and the performance indicator is the energy consumption. As frequency tuning control in real-life applications is still a major challenge at the forefront of research in energy harvesting [13], the tuning control was omitted from the simulation model for real vibration input and only used for the sine wave input.

Fig. 7 shows the photo of a hardware prototype that we have built and tested. The parameter values of microgenerator in the prototype harvester are listed in Table III. The prototype uses a bridge rectifier as the power processing circuit, a $0.55 \mathrm{~F}$ supercapacitor, model GS206 from CAP-XX, which powers a eZ430-RF2500 wireless sensor node from Texas Instruments. The on-board MSP430F2274 microcontroller of the sensor node was programmed to execute the data transmission scenario defined in Table II. The maximum RSM estimation errors for the prototype did not exceed $8.4 \%$ for the sine wave vibrations and $6.1 \%$ for the ferry boat engine vibrations.

Table IV summarizes the design parameters and performance indicators. 
TABLE III

NUMERICAL VALUES OF MICROGENERATOR PARAMETERS

\begin{tabular}{|l|l|l||l|l|l|}
\hline Symbol & Value & Unit & Symbol & Value & Unit \\
\hline$m$ & $2.4 \mathrm{e}-3$ & $\mathrm{~kg}$ & $l$ & $1.3 \mathrm{e}-3$ & $\mathrm{~m}$ \\
$c_{p}$ & $1.3 \mathrm{e}-3$ & $\mathrm{Nm}^{-1} \mathrm{~s}^{-1}$ & $R_{c}$ & 4500 & $\Omega$ \\
$N$ & 6000 & & $L_{c}$ & 0.58 & $\mathrm{H}$ \\
$B$ & 0.45 & $\mathrm{~T}$ & $F_{b}$ & 0.5 & $\mathrm{~N}$ \\
\hline
\end{tabular}

TABLE IV

SUMMARY OF THE DESIGN PARAMETERS AND PERFORMANCE INDICATORS INCLUDED IN THE DESIGN EXPLORATION TOOL.

\begin{tabular}{|c|c|c|c|}
\hline & $\begin{array}{l}\text { Design } \\
\text { paramete }\end{array}$ & & $\begin{array}{l}\text { Performance } \\
\text { indicators }\end{array}$ \\
\hline \multirow{5}{*}{$\begin{array}{l}\text { Vibration } \\
\text { source }\end{array}$} & \multirow{3}{*}{$\begin{array}{l}\text { Sine } \\
\text { wave }\end{array}$} & Amplitude $(A)$ & \\
\hline & & Time between & \\
\hline & & frequency changes $\left(T_{f}\right)$ & \\
\hline & \multicolumn{2}{|c|}{ Real vibration from ferry } & \\
\hline & \multicolumn{2}{|c|}{ Real vibration from car engine } & \\
\hline \multirow{3}{*}{$\begin{array}{l}\text { Micro- } \\
\text { generator }\end{array}$} & \multicolumn{2}{|c|}{ Proof mass $(M)$} & Generated power \\
\hline & \multicolumn{2}{|c|}{ Number of coil turns $(N)$} & Maximum \\
\hline & \multicolumn{2}{|c|}{ Mechanical Q factor $(Q)$} & displacement \\
\hline \multirow{5}{*}{$\begin{array}{l}\text { Power } \\
\text { processing }\end{array}$} & \multicolumn{2}{|c|}{ Bridge rectifier } & Power consumntion \\
\hline & \multicolumn{2}{|c|}{ 4-stage VM } & Power consumpinon \\
\hline & \multirow{3}{*}{$\begin{array}{l}\text { Boost } \\
\text { converter }\end{array}$} & Inductor value $(L)$ & \\
\hline & & Switching Frequency $\left(F_{s}\right)$ & Transfer efficiency \\
\hline & & Switching duty cycle $\left(D_{s}\right)$ & \\
\hline Storage & \multicolumn{2}{|c|}{ Super-capacitor value $(C)$} & Super-cap voltage \\
\hline $\begin{array}{l}\text { Sensor } \\
\text { node }\end{array}$ & \multicolumn{2}{|c|}{$\begin{array}{l}\text { Time interval between } \\
\text { transmissions }\left(T_{r}\right)\end{array}$} & $\begin{array}{l}\text { Number of } \\
\text { transmissions }\end{array}$ \\
\hline $\begin{array}{l}\text { Tuning } \\
\text { control }\end{array}$ & \multicolumn{2}{|c|}{$\begin{array}{l}\text { Tuning controller duty cycle }\left(D_{t}\right) \\
\text { (not for real vibration input) }\end{array}$} & Power consumption \\
\hline
\end{tabular}

\section{RESULTS AND DISCUSSION}

The design exploration tool enables the user to change the design parameters of a system and to get the performance indicators instantly, as shown in Table V. The CPU times shown in the table illustrate the massive acceleration achieved by the proposed technique of fast design space exploration. Results were obtained for several test scenarios. Table $\mathrm{V}$ also lists the number of parameters for each model and the number of simulations needed to build the RSM. As the RSM build process is not interactive, parallel simulations were carried out by Southampton University's high performance computing cluster [14] to obtain the required RSM.

The significance of the prosed technique lies mainly in its capability to permit the designer to look for interesting properties in the design by a quick investigation of a large number of possible tradeoffs between the design parameters and performance indicators. The best selected designs may need to be checked for the performance prediction accuracy by running full HDL simulations.

\section{A. Effect of coil turns on output power}

For an electromagnetic microgenerator, more coil turns lead to higher generated energy within the coils. However, due to the internal resistance of the coil, more coil turns also mean higher internal resistance and hence more internal loss. The microgenerator generates maximum output power when the internal resistance matches the external resistance [1]. For a specific power processing circuit, the design exploration tool can find the optimal number of coil turns of the energy harvester. Fig. 9 shows the microgenerator output power versus number of coil turns for three different power processing circuits where all the other design parameters are fixed.

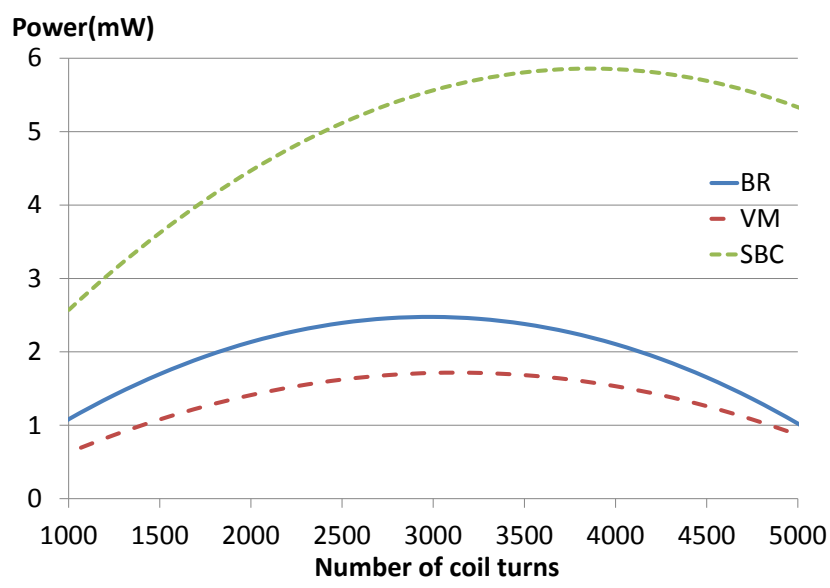

Fig. 9. Microgenerator output power vs. number of coil turns (Ferry vibration, $\left.M=5 \mathrm{~g}, Q=100, C=0.1 \mathrm{~F}, T_{r}=1 \mathrm{sec}\right)$.

\section{B. Effect of switching frequency on SBC's performance}

The inductor value, the switching frequency and the duty cycle determine the performance of a switching boost converter. Fig. 10 shows the SBC's transfer efficiency and power delivered to the supercapacitor, i.e the useful power, plotted against the switching frequency. All the other design parameters are the same. For validation, a number of design points are simulated and the results are plotted as dots on Fig. 10. It can be seen that the difference between estimated and simulated values are generally less that $10 \%$. Also note that the best transfer efficiency does not necessarily leads to the highest useful power.

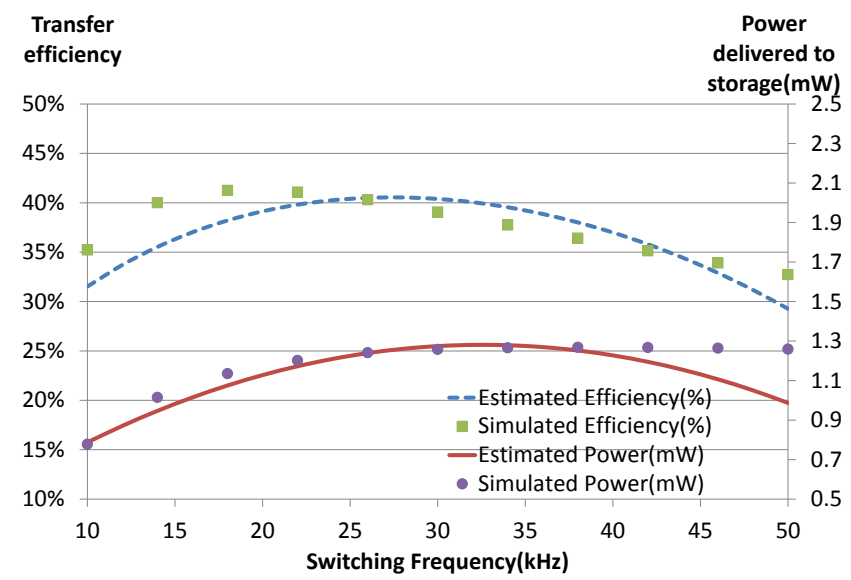

Fig. 10. Transfer efficiency and delivered power vs. switching frequency (Car vibration, $M=10 \mathrm{~g}, N=3000, Q=200, C=0.55 \mathrm{~F}, T_{r}=30 \mathrm{sec}, L=5 \mathrm{mH}$, $D_{s}=50 \%$ ).

\section{Effect of tuning duty cycle on number of transmissions}

The tuning controller wakes up periodically to check if the microgenerator's resonant frequency matches the input fre- 
TABLE V

CPU TIMES OF THE HDL MODEL SIMULATION COMPARING TO THE RSM MODEL ESTIMATION

\begin{tabular}{|c|c|c|c|c|c|c|}
\hline $\begin{array}{l}\text { Vibration } \\
\text { Source }\end{array}$ & $\begin{array}{l}\text { Power } \\
\text { processing }\end{array}$ & $\begin{array}{l}\text { CPU time of one } \\
\text { simulation }\end{array}$ & $\begin{array}{l}\text { No of parameters } \\
\text { (parameters included) }\end{array}$ & $\begin{array}{l}\text { No of simulations } \\
\text { to build RSM }\end{array}$ & $\begin{array}{l}\text { CPU time of } \\
\text { RSM estimation }\end{array}$ & $\begin{array}{l}\text { Average error of } \\
\text { RSM estimation }\end{array}$ \\
\hline \multirow{3}{*}{$\begin{array}{l}\text { Sine } \\
\text { wave }\end{array}$} & $\mathrm{BR}$ & 0 hours 46 mins & $8\left(A, T_{f}, M, N, Q, C, T_{r}, D_{t}\right)$ & 45 & \multirow{9}{*}{$25 \mu \mathrm{s}$} & $16.2 \%$ \\
\hline & VM & 0 hours 50 mins & $8\left(A, T_{f}, M, N, Q, C, T_{r}, D_{t}\right)$ & 45 & & $12.6 \%$ \\
\hline & SBC & 13 hours $21 \mathrm{mins}$ & $11\left(A, T_{f}, M, N, Q, L, F_{s}, D_{s}, C, T_{r}, D_{t}\right)$ & 78 & & $14.0 \%$ \\
\hline \multirow{3}{*}{$\begin{array}{l}\text { Ferry } \\
\text { vibration }\end{array}$} & $\overline{B R}$ & 1 hours 5 mins & $5\left(M, N, Q, C, T_{r}\right)$ & 21 & & $10.8 \%$ \\
\hline & VM & 1 hours 14 mins & $5\left(M, N, Q, C, T_{r}\right)$ & 21 & & $11.1 \%$ \\
\hline & SBC & 4 hours 52 mins & $8\left(M, N, Q, L, F_{s}, D_{s}, C, T_{r}\right)$ & 45 & & $13.4 \%$ \\
\hline \multirow{3}{*}{$\begin{array}{l}\text { Car } \\
\text { vibration }\end{array}$} & BR & 0 hours $33 \mathrm{mins}$ & $5\left(M, N, Q, C, T_{r}\right)$ & 21 & & $6.4 \%$ \\
\hline & VM & 0 hours $37 \mathrm{mins}$ & $5\left(M, N, Q, C, T_{r}\right)$ & 21 & & $7.2 \%$ \\
\hline & SBC & 2 hours 19 mins & $8\left(M, N, Q, L, F_{s}, D_{s}, C, T_{r}\right)$ & 45 & & $14.7 \%$ \\
\hline
\end{tabular}

quency. As the tuning controller consumes energy when wake up, its duty cycle needs to be chosen carefully according to the input vibration. A short duty cycle can detect the frequency change quickly but may consume energy unnecessarily. A long duty cycle can save energy of the tuning controller but the generated energy from the microgenerator will drop dramatically when there is a difference between the input frequency and the resonant frequency. Fig. 11 shows the number of transmissions per minute plotted against both the tuning duty cycle and the time between frequency changes. It can be seen that the tuning duty cycle can greatly affect the system performance. For the fixed time between frequency change, there are two good values for the tuning duty cycle. This is because the total number of transmissions can be increased by either tuning frequently to match the input frequency change or by tuning less frequently to save energy on tuning. For the fixed tuning duty cycle, the number of transmissions increase from left to right. This is due to the longer time between frequency changes, the less tuning needed.

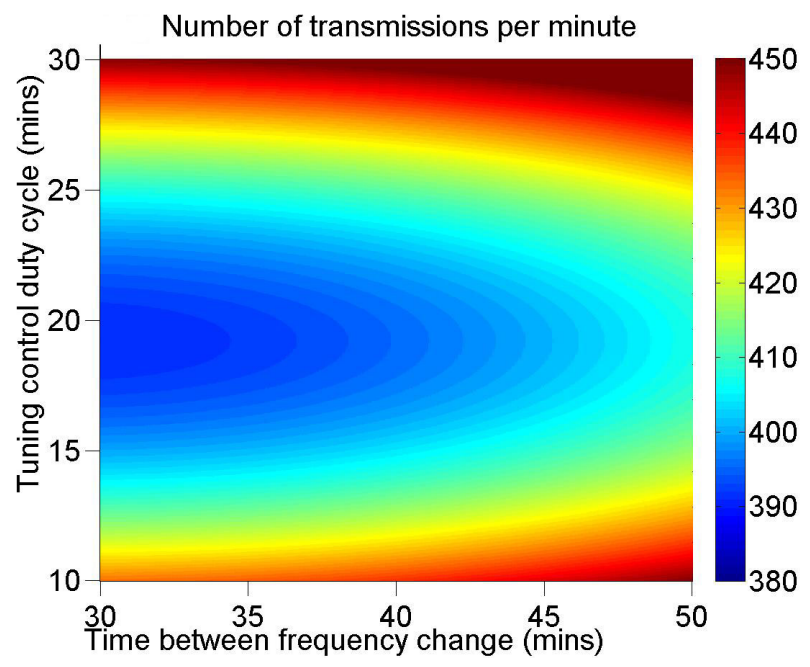

Fig. 11. Number of transmissions per minute vs. tuning duty cycle and time between frequency changes (Sine wave vibration, VM power processing circuit, $\left.A=750 \mathrm{mg}, M=10 \mathrm{~g}, N=3000, Q=200, C=0.55 \mathrm{~F}, T_{r}=1 \mathrm{sec}\right)$.

\section{CONCLUSION}

This paper highlights the benefits of fast design space exploration in the design of energy-harvester-powered wireless sensor nodes. The identified design parameters are investigated using the RSM based technique. We use SystemC-A to model the system and developed a toolkit to demonstrate the effectiveness of our approach.

As pointed out in the discussion above, the sets of results obtained in the test scenarios show the power of the RSM technique in exploring the designs, where essential knowledge about the interaction between design parameters and performance can be obtained near-instantly, without costly simulations. Future work will focus on the optimization of the RSM models so that designers can be guided by the tool in searching the areas in the design space where one should look for systems exhibiting the best performance.

\section{ACKNOWLEDGMENT}

This work was supported by the Engineering and Physical Sciences Research Council (EPSRC), UK under grant number EP/G067740/1 "Next Generation Energy-Harvesting Electronics: Holistic Approach," website: (http://www.holistic.ecs.soton.ac.uk).

\section{REFERENCES}

[1] S. Roundy, P. K. Wright, and J. M. Rabaey, Energy scavenging for wireless sensor networks: with special focus on vibrations. Springer, 2004.

[2] C. Alippi, R. Camplani, C. Galperti, and M. Roveri, "A robust, adaptive, solar-powered WSN framework for aquatic environmental monitoring," IEEE Sensors Journal, vol. 11, no. 1, pp. 45-55, 2011.

[3] Q. Ling, Z. Tian, Y. Yin, and Y. Li, "Localized structural health monitoring using energy-efficient wireless sensor networks," IEEE Sensors Journal, vol. 9, no. 11, pp. 1596-1604, 2009.

[4] A. Sapio and G. Tsouri, "Low-power body sensor network for wireless ECG based on relaying of creeping waves at $2.4 \mathrm{GHz}$," in International Conference on Body Sensor Networks (BSN), 2010, pp. 167-173.

[5] T. Kazmierski, G. Merrett, L. Wang, B. Al-Hashimi, A. Weddell, and I. Ayala Garcia, "Modeling of wireless sensor nodes powered by tunable energy harvesters: Hdl-based approach," IEEE Sensors Journal, vol. 12, no. 8, pp. 2680-2689, 2012.

[6] H. Boussetta, M. Marzencki, S. Basrour, and A. Soudani, "Efficient physical modeling of MEMS energy harvesting devices with VHDL-AMS," IEEE Sensors Journal, vol. 10, no. 9, pp. 1427-1437, 2010.

[7] T. Kazmierski, L. Wang, B. Al-Hashimi, and G. Merrett, "An explicit linearized state-space technique for accelerated simulation of electromagnetic vibration energy harvesters," IEEE Transactions on Computer-Aided Design of Integrated Circuits and Systems, vol. 31, no. 4, pp. 522-531, 2012.

[8] R. Fisher, J. Bennet, and Y. F., Statistical Methods Experimental Design and Scientific Interference. Oxford University Press, USA, 1990.

[9] J. Jacquez, "Design of experiments," Journal of the Franklin Institute, vol. 335, no. 2, pp. 259-279, 1998. 
[10] D. Zhu, S. Roberts, J. Tudor, and S. Beeby, "Design and experimental characterization of a tunable vibration-based electromagnetic micro-generator," Sensors and Actuators A: Physical, vol. 158, no. 2, pp. 284-293, 2010.

[11] G. Akoun and J. Yonnet, "3D analytical calculation of the forces exerted between two cuboidal magnets," IEEE Transaction on Magnetics, vol. 20, no. 5, pp. 1962-1964, 1984.

[12] EH Network Data Repository, August, 2012. [Online]. Available: http://eh-network.org/data/

[13] A. Weddell, G. Merrett, S. Barrow, and B. Al-Hashimi, "Vibration-powered sensing system for engine condition monitoring," in Wireless Sensor Systems (WSS 2012), IET Conference on, 18-19 June 2012, pp. 1-5.

[14] The Iridis Compute Cluster, August, 2012. [Online]. Available: http://www.southampton.ac.uk/isolutions/computing/hpc/iridis/ 